\title{
Pulmonary disease as a risk factor for transfusion-related acute lung injury
}

\author{
Akira Yokoyama $^{1}$, Yukiyo Sakamoto ${ }^{1}$, Taisuke Jo $\mathbb{1}^{1,2}$, Hirokazu Urushiyama ${ }^{1}$, Hiroyuki Tamiya ${ }^{1}$, \\ Goh Tanaka ${ }^{1}$, Hiroki Matsui ${ }^{3}$, Kiyohide Fushimi ${ }^{4}$, Hideo Yasunaga ${ }^{3}$ and Takahide Nagase ${ }^{1}$
}

${ }^{1}$ Dept of Respiratory Medicine, Graduate School of Medicine, The University of Tokyo, Tokyo, Japan. ${ }^{2}$ Dept of Health Services Research, Graduate School of Medicine, The University of Tokyo, Tokyo, Japan. ${ }^{3}$ Dept of Clinical Epidemiology and Health Economics, School of Public Health, The University of Tokyo, Tokyo, Japan. ${ }^{4}$ Dept of Health Policy and Informatics, Tokyo Medical and Dental University Graduate School of Medicine, Tokyo, Japan.

Corresponding author: Taisuke Jo (jo-taisuke@umin.ac.jp)

Shareable abstract (@ERSpublications)

Physicians should be aware of the risk of developing transfusion-related acute lung injury when performing a blood transfusion in patients with interstitial lung disease. https://bit.ly/3jdoZQP

Cite this article as: Yokoyama A, Sakamoto Y, Jo T, et al. Pulmonary disease as a risk factor for transfusionrelated acute lung injury. ERJ Open Res 2021; 7: 00039-2021 [DOI: 10.1183/23120541.00039-2021].

Copyright @The authors 2021

This version is distributed under the terms of the Creative Commons Attribution NonCommercial Licence 4.0. For commercial reproduction rights and permissions contact permissions@ersnet.org

Received: 18 Jan 2021

Accepted: 20 June 2021

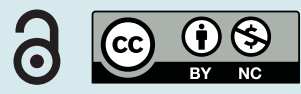

\section{Abstract}

Transfusion-related acute lung injury (TRALI) is a severe condition characterised by noncardiogenic pulmonary oedema that develops within $6 \mathrm{~h}$ of blood transfusion. Patient factors and blood products have both been implicated in the development of TRALI; however, the role of pulmonary disease has not been investigated. We aimed to determine whether pulmonary disease is a risk factor for TRALI.

We conducted a nested case-control study using data from the Diagnosis Procedure Combination database, a nationwide inpatient database in Japan, between July 2010 and March 2015. Case patients who developed TRALI were 1:4-matched with control patients for sex, age and same hospital for receipt of blood transfusion. We conducted a multivariable conditional logistic regression analysis to evaluate the associations of TRALI with various factors including comorbidities, body mass index (BMI) and plasmacontaining blood products.

We identified 2019501 hospitalised patients who received a blood transfusion. Among these patients, 72 developed TRALI. The 72 case patients had higher proportions of haematological malignancy, trauma and interstitial lung disease (ILD) than the 288 matched control patients. The multivariable conditional logistic regression analysis showed that occurrence of TRALI was associated with ILD (odds ratio, 3.88; 95\% confidence interval, $1.11-13.6)$, BMI $\geqslant 25.0 \mathrm{~kg} \cdot \mathrm{m}^{-2}(2.10 ; 1.05-4.24)$ and plasma-containing blood products $(1.94 ; 1.10-3.42)$, but not with infectious lung disease or obstructive airway disease.

In conclusion, ILD was an independent risk factor for the development of TRALI. Physicians should be aware of the increased risk of TRALI in patients with ILD.

\section{Introduction}

Transfusion-related acute lung injury (TRALI) is one of the adverse events associated with blood transfusion and is characterised by noncardiogenic pulmonary oedema that develops within $6 \mathrm{~h}$ of blood transfusion [1]. The proportions of patients who developed TRALI were reported to vary from $0.08 \%$ to 15.0\% [2]. Previous studies identified several risk factors for TRALI, including sepsis, chronic alcoholism and surgery for liver transplantation [3-5]. Anti-white blood cell antibody was identified as another risk factor for the development of TRALI [5]. Efforts were thus undertaken to reduce the risk of TRALI by avoiding the use of blood donated by women, especially pregnant women [6].

Regarding the underlying mechanism for TRALI, a two-event model has been proposed [7]. In this model, both patient factors and blood products are involved in the development of TRALI. The first event is related to patient clinical conditions that lead to activation of the pulmonary endothelium by polymorphonuclear leukocytes (PMNs). This process occurs when PMNs become primed and functionally hyperactive due to the background disease. The second event is related to infusion of blood products 
containing specific antibodies or mediators that affect PMNs. As a result, activated PMNs cause endothelial damage and capillary leakage leading to the development of TRALI. Pulmonary diseases (infectious lung disease, obstructive airway disease, and interstitial lung disease (ILD)) can activate endothelial cells via PMNs [8].

Acute and severe deterioration of the lungs can lead to fatal outcomes in patients with pulmonary disease. Therefore, it is essential to determine whether pulmonary disease is a risk factor for TRALI. However, no studies to date have evaluated the risk of TRALI in patients with pulmonary disease. In the present study, we aimed to determine whether pulmonary disease was a risk factor for TRALI in patients who required blood transfusion by conducting a nested case-control study using a Japanese inpatient database.

Methods

\section{Data source}

We conducted a nested case-control study using data from the Diagnosis Procedure Combination database, a nationwide inpatient database in Japan, between July 2010 and March 2015. The database includes data for more than 1000 hospitals in Japan, and covers more than $90 \%$ of all tertiary-care emergency hospitals. The number of hospitalised patients in participating hospitals reaches about 7 million per year, comprising more than $50 \%$ of all hospitalised patients in Japan [9].

The database includes data on patient age, sex, body height and weight, smoking history, main diagnoses, comorbidities on admission, complications occurring during hospitalisation, discharge status, medications, and treatments. The study was approved by the Institutional Review Board of The University of Tokyo. The board waived the requirement for informed patient consent because of the anonymous nature of the data.

\section{Patient selection}

We identified patients who were discharged during the study period and who received blood products for transfusion (whole blood, red blood cells, platelets, or fresh-frozen plasma), but not autologous blood transfusion, during hospitalisation. We used the International Statistical Classification of Diseases and Related Health Problems, 10th revision (ICD-10) code for TRALI (J708) to identify case patients who developed TRALI.

\section{Patient characteristics and blood products}

The following patient characteristics were collected: sex; age; body mass index (BMI); smoking history; comorbidities; and blood products used for transfusion. Patients were categorised into four age groups ( $<39,40-59,60-79$ and $\geqslant 80$ years). BMI $\left(\mathrm{kg} \cdot \mathrm{m}^{-2}\right)$ was categorised into four groups $(<18.5,18.5-24.9$, $\geqslant 25$ and missing). Comorbidities included: haematopoietic disorder (ICD-10 codes, D50-D77); haematological malignancy (C81-C96); other malignancy (C00-C80, C97-D48); trauma (S00-T98); gastroenterological disorder (K00-K93); sepsis (A400-A403, A408-A415, A418, A419); hepatic dysfunction including liver disease (K70-K77), alcoholism (F101, F102, F105, F107, G312, K701, K703, K704, K709, K852, K860) and liver surgery; heart failure (I20-I25, I34-I37, I42, I43, I48, I50); infectious lung disease (J09-J22, J85, J86); obstructive airway disease (J40-J47); and ILD (J80-J82, J84, J990, J991). Obstructive airway disease included chronic obstructive pulmonary disease, chronic bronchitis, bronchial asthma and bronchiectasis.

Blood products included whole blood, red blood cells, platelets and fresh-frozen plasma. All of these blood products except for red blood cells were grouped into plasma-containing products.

\section{Treatments and outcomes}

We evaluated the frequencies of intensive care unit (ICU) admission, emergency intubation, systemic corticosteroid administration and use of sivelestat after initial blood transfusion during hospitalisation in case patients and control patients to better understand the disease severity in the examined patients. We evaluated all-cause in-hospital mortality because the database did not provide data on causes of death.

\section{Statistical analysis}

For this study, a nested case-control design was adopted to evaluate risk factors for the development of TRALI. Case patients who developed TRALI were 1:4-matched with control patients for sex, age and same hospital for receipt of blood transfusion. Sex and age were matched because they are variables associated with both the exposure (lung disease) and the disease (TRALI) and are commonly used for matching. Hospitals were matched because individual hospitals may have different practices for lung disease, transfusion and diagnosis of TRALI. We considered these three variables to be strong confounders and therefore used them for case-control matching. We matched four control patients to one case patient to 
preserve the control patients and increase the sample size, which can improve the statistical power and the precision of the findings [10]. Patient characteristics were compared using the chi-square test for binominal and categorical variables. The Mann-Whitney $U$ test or the t-test were used to compare numerical variables, depending on the distribution of each variable. We conducted a multivariable conditional logistic regression analysis to evaluate risk factors for TRALI. Independent variables included potential risk factors based on previous studies (sepsis, liver dysfunction), pulmonary disease (infectious lung disease, obstructive airway disease, ILD), other comorbidity (heart failure) and BMI. We also included plasma-containing products in the independent variables. Independent variables were chosen from risk factors that were identified in previous studies and available in the Diagnosis Procedure Combination database. Variance inflation factors were estimated for the variables used in the model and checked for multicollinearity. The results derived from the multivariable model were shown as odds ratio, 95\% confidence interval and $\mathrm{p}$-value for each independent variable. Values of $\mathrm{p}<0.05$ were considered significant. All analyses were conducted using SPSS version 23.0 (IBM SPSS, Armonk, NY) or Stata version 16.0 (StataCorp, College Station, TX).

\section{Results}

\section{Patient characteristics and blood products}

The patient characteristics are shown in table 1. We identified 2019501 hospitalised patients who received a blood transfusion. Among these patients, 72 developed TRALI (38 men, 34 women; mean age \pm standard deviation, $69.4 \pm 2.03$ years). The 72 case patients were 1:4-matched with 288 control patients who received a blood transfusion during hospitalisation but were not diagnosed as TRALI. No significant differences were observed in sex and age distribution between the two groups. A higher proportion of patients with $\mathrm{BMI} \geqslant 25 \mathrm{~kg} \cdot \mathrm{m}^{-2}$ was observed in the case patients compared with the control patients after matching (25.0\% versus $14.6 \%$ ). Patients with smoking history did not differ significantly between the case patients and control patients after matching $(37.5 \%$ versus $39.6 \%, \mathrm{p}=0.746)$. Regarding comorbidities, the case patients had higher proportions of haematological malignancy, trauma and ILD than the control patients.

The blood products used for blood transfusion are shown in table 2. Only a very small fraction of patients received whole blood for transfusion. Red blood cells were most commonly used in both the case patients

TABLE 1 Characteristics and comorbidities of patients with and without transfusion-related acute lung injury (TRALI)

\begin{tabular}{|c|c|c|c|c|c|c|c|c|c|c|}
\hline \multirow[t]{3}{*}{ Characteristics } & \multicolumn{5}{|c|}{ All } & \multicolumn{5}{|c|}{ After matching } \\
\hline & \multicolumn{2}{|c|}{$\begin{array}{c}\text { Control } \\
(n=2019501)\end{array}$} & \multicolumn{2}{|c|}{ TRALI $(n=72)$} & \multirow[b]{2}{*}{$\mathrm{p}$-value } & \multicolumn{2}{|c|}{$\begin{array}{l}\text { Control } \\
(n=288)\end{array}$} & \multicolumn{2}{|c|}{ TRALI $(n=72)$} & \multirow[b]{2}{*}{$\mathrm{p}$-value } \\
\hline & $\mathrm{n}$ & $\%$ & $\mathrm{n}$ & $\%$ & & $\mathrm{n}$ & $\%$ & $\mathrm{n}$ & $\%$ & \\
\hline Sex (male) & 1052795 & 52.1 & 38 & 52.8 & 0.913 & 152 & 52.8 & 38 & 52.8 & 1.000 \\
\hline Age (years) & & & & & 0.854 & & & & & 0.774 \\
\hline$<39$ & 135759 & 6.72 & 4 & 5.56 & & 16 & 5.56 & 4 & 5.56 & \\
\hline $40-59$ & 258474 & 12.8 & 8 & 11.1 & & 34 & 11.8 & 8 & 11.1 & \\
\hline $60-79$ & 992584 & 49.2 & 39 & 54.2 & & 171 & 59.4 & 39 & 54.2 & \\
\hline$\geqslant 80$ & 632612 & 31.3 & 21 & 29.2 & & 67 & 23.3 & 21 & 29.2 & \\
\hline Body mass index $\left(\mathrm{kg} \cdot \mathrm{m}^{-2}\right)$ & & & & & 0.131 & & & & & 0.161 \\
\hline$<18.5$ & 1103086 & 54.6 & 36 & 50.0 & & 167 & 58.0 & 36 & 50.0 & \\
\hline $18.5-24.9$ & 429990 & 21.3 & 12 & 16.7 & & 60 & 20.8 & 12 & 16.7 & \\
\hline$\geqslant 25$ & 306249 & 15.2 & 18 & 25.0 & & 42 & 14.6 & 18 & 25.0 & \\
\hline Missing & 180104 & 8.92 & 6 & 8.33 & & 19 & 6.60 & 6 & 8.33 & \\
\hline Smoking history & 774301 & 38.3 & 27 & 37.5 & 0.978 & 114 & 39.6 & 27 & 37.5 & 0.746 \\
\hline \multicolumn{11}{|l|}{ Comorbidities } \\
\hline Haematopoietic disorder & 724501 & 35.9 & 32 & 44.4 & 0.130 & 108 & 37.5 & 32 & 44.4 & 0.280 \\
\hline Haematological malignancy & 182971 & 9.1 & 12 & 16.7 & 0.025 & 24 & 8.3 & 12 & 16.7 & 0.035 \\
\hline Other malignancy & 718948 & 35.6 & 26 & 36.1 & 0.928 & 110 & 38.2 & 26 & 36.1 & 0.744 \\
\hline Trauma & 302636 & 15.0 & 24 & 33.3 & $<0.001$ & 42 & 14.6 & 24 & 33.3 & $<0.001$ \\
\hline Gastroenterological disorder & 824421 & 40.8 & 16 & 22.2 & 0.001 & 128 & 44.4 & 16 & 22.2 & 0.001 \\
\hline Heart failure & 486578 & 24.1 & 21 & 29.2 & 0.314 & 66 & 22.9 & 21 & 29.2 & 0.268 \\
\hline Liver dysfunction & 135856 & 6.7 & 5 & 6.9 & 0.941 & 24 & 8.3 & 5 & 6.9 & 0.699 \\
\hline Infectious lung disease & 86310 & 4.3 & 6 & 8.3 & 0.089 & 15 & 5.2 & 6 & 8.3 & 0.312 \\
\hline Interstitial lung disease & 22049 & 1.1 & 5 & 6.9 & $<0.001$ & 6 & 2.1 & 5 & 6.9 & 0.032 \\
\hline Obstructive airway disease & 71602 & 3.6 & 4 & 5.6 & 0.356 & 13 & 4.5 & 4 & 5.6 & 0.709 \\
\hline Sepsis & 94048 & 4.7 & 5 & 6.9 & 0.357 & 17 & 5.9 & 5 & 6.9 & 0.741 \\
\hline
\end{tabular}


TABLE 2 Blood products used for transfusion

\begin{tabular}{|c|c|c|c|c|c|c|c|c|c|c|}
\hline & \multicolumn{5}{|c|}{ All } & \multicolumn{5}{|c|}{ After matching } \\
\hline & \multicolumn{2}{|c|}{$\begin{array}{c}\text { Control } \\
(n=2019501)\end{array}$} & \multicolumn{2}{|c|}{ TRALI $(n=72)$} & \multirow[b]{2}{*}{ p-value } & \multicolumn{2}{|c|}{$\begin{array}{l}\text { Control } \\
(n=288)\end{array}$} & \multicolumn{2}{|c|}{ TRALI $(n=72)$} & \multirow[b]{2}{*}{ p-value } \\
\hline & $\mathrm{n}$ & $\%$ & $\mathrm{n}$ & $\%$ & & $\mathrm{n}$ & $\%$ & $\mathrm{n}$ & $\%$ & \\
\hline Red blood cells & 1881694 & 93.2 & 71 & 98.6 & 0.068 & 273 & 94.8 & 71 & 98.6 & 0.160 \\
\hline Plasma-containing products & 673202 & 33.3 & 40 & 55.6 & $<0.001$ & 115 & 39.9 & 40 & 55.6 & 0.017 \\
\hline Whole blood & 1153 & 0.1 & 0 & 0 & 0.839 & 0 & 0 & 0 & 0 & \\
\hline Platelets & 424662 & 21.0 & 28 & 38.9 & $<0.001$ & 61 & 21.2 & 28 & 38.9 & 0.002 \\
\hline Fresh-frozen plasma & 412556 & 20.4 & 30 & 41.7 & $<0.001$ & 86 & 29.9 & 30 & 41.7 & 0.055 \\
\hline
\end{tabular}

and control patients, with no significant difference. Platelets and fresh-frozen plasma were used more often in the case patients than in the control patients (38.9\% versus $21.2 \%$ and $41.7 \%$ versus $29.9 \%$, respectively). As a result, the proportion of patients who received plasma-containing products was significantly higher in the case patients compared with the control patients $(55.6 \%$ versus $39.9 \%$, $\mathrm{p}=0.017)$.

\section{Treatments and outcomes}

The frequencies of the treatments required after initial blood transfusion during hospitalisation are shown in table 3. After matching, the case patients were more likely to undergo ICU admission, receive emergency intubation, require systemic corticosteroid administration and use sivelestat. In-hospital mortality was not significantly higher in the case patients than in the control patients $(20.8 \%$ versus $16.7 \%$, $\mathrm{p}=0.405)$.

\section{Multivariable conditional regression model}

The results of the multivariable conditional regression model are shown in table 4. Occurrence of TRALI was significantly associated with ILD (odds ratio, 3.88; 95\% confidence interval, 1.11-13.6), BMI $\geqslant 25.0 \mathrm{~kg} \cdot \mathrm{m}^{-2}(2.10 ; 1.05-4.24)$ and plasma-containing blood products $(1.94 ; 1.10-3.42)$, but was not significantly associated with infectious lung disease or obstructive airway disease. The variance inflation factors for all variables were less than two, which indicates that multicollinearity is unlikely to exist among the variables.

\section{Discussion}

We evaluated three pulmonary disease categories (infectious lung disease, obstructive airway disease and ILD) as potential risk factors for the development of TRALI. Of these, only ILD was significantly associated with the development of TRALI.

TABLE 3 In-hospital treatments and outcomes of patients with or without transfusion-related acute lung injury (TRALI)

\begin{tabular}{|c|c|c|c|c|c|c|c|c|c|c|}
\hline & \multicolumn{5}{|c|}{ All } & \multicolumn{5}{|c|}{ After matching } \\
\hline & \multicolumn{2}{|c|}{$\begin{array}{c}\text { Control } \\
(n=2019501)\end{array}$} & \multicolumn{2}{|c|}{ TRALI $(n=72)$} & \multirow[b]{2}{*}{ p-value } & \multicolumn{2}{|c|}{$\begin{array}{l}\text { Control } \\
(n=288)\end{array}$} & \multicolumn{2}{|c|}{ TRALI $(n=72)$} & \multirow[b]{2}{*}{$\mathrm{p}$-value } \\
\hline & $\mathrm{n}$ & $\%$ & $\mathrm{n}$ & $\%$ & & $\mathrm{n}$ & $\%$ & $\mathrm{n}$ & $\%$ & \\
\hline \multicolumn{11}{|l|}{ Treatments } \\
\hline Intensive care unit admission & 278920 & 13.8 & 33 & 45.8 & $<0.001$ & 65 & 22.6 & 33 & 45.8 & $<0.001$ \\
\hline Emergency intubation & 91180 & 4.5 & 19 & 26.4 & $<0.001$ & 24 & 8.3 & 19 & 26.4 & $<0.001$ \\
\hline Corticosteroid & 411378 & 20.4 & 56 & 77.8 & $<0.001$ & 102 & 35.4 & 56 & 77.8 & $<0.001$ \\
\hline Sivelestat & 55421 & 2.7 & 26 & 36.1 & $<0.001$ & 13 & 4.5 & 26 & 36.1 & $<0.001$ \\
\hline \multicolumn{11}{|l|}{ Outcomes } \\
\hline All-cause in-hospital mortality & 313253 & 15.5 & 15 & 20.8 & 0.212 & 48 & 16.7 & 15 & 20.8 & 0.405 \\
\hline
\end{tabular}




\begin{tabular}{|c|c|c|c|}
\hline & Odds ratio & 95\% Confidence interval & p-value \\
\hline Plasma-containing blood products & 1.94 & $1.10-3.42$ & 0.022 \\
\hline \multicolumn{4}{|l|}{ Body mass index $\left(\mathrm{kg} \cdot \mathrm{m}^{-2}\right)$} \\
\hline$<18.5$ & 0.84 & $0.39-1.80$ & 0.655 \\
\hline $18.5-24.9$ & Reference & & \\
\hline$\geqslant 25.0$ & 2.10 & $1.05-4.24$ & 0.037 \\
\hline Missing & 1.71 & $0.57-5.10$ & 0.336 \\
\hline Sepsis & 0.81 & $0.25-2.62$ & 0.727 \\
\hline Liver dysfunction & 0.79 & $0.27-2.36$ & 0.678 \\
\hline Heart failure & 1.49 & $0.80-2.79$ & 0.212 \\
\hline Infectious lung disease & 1.87 & $0.65-5.44$ & 0.248 \\
\hline Obstructive airway disease & 1.32 & $0.39-4.51$ & 0.656 \\
\hline Interstitial lung disease & 3.88 & $1.11-13.61$ & 0.034 \\
\hline
\end{tabular}

In our cohort, the proportion of patients who developed TRALI was as low as $0.0036 \%$. The reported incidence of TRALI has varied widely $(0.01 \%-5.1 \%)$ among previous studies because they had different study populations $[4,11,12]$. The occurrence of TRALI in our real-world database study was much lower compared with these previous studies. There are several possible explanations for this discrepancy. Firstly, TRALI may have been underdiagnosed because no biomarkers have been identified to assist with its diagnosis. Consequently, it can be difficult to distinguish TRALI from other diseases that resemble TRALI, such as transfusion-associated circulatory overload (TACO), acute respiratory distress syndrome (ARDS) and acute exacerbation of ILD. Secondly, there may be differences in the blood products used for transfusion. In Japan, blood donation during pregnancy and until 6 months after delivery is prohibited, mainly from the viewpoint of safety during maternity care. The percentage of male-derived fresh-frozen plasma production reached almost 100\% in April 2011 [13]. Blood products from women, particularly pregnant women, have a higher prevalence of anti- human leukocyte antigen (HLA) antibodies that can increase the incidence of TRALI. The male-predominant plasma transfusion strategy in Japan may have successfully mitigated the impact of donor anti-HLA antibodies. In fact, the cumulative numbers of patients with TRALI, possible TRALI and TACO from 2010 to 2015 reported by the Japan Red Cross Society were 47, 52 and 162, respectively [14]. Given that the database used in the present study covers $50 \%$ of all hospitalised patients in Japan, the number of patients with TRALI captured in our study may be reasonable.

The all-cause in-hospital mortality in patients with TRALI was $20.8 \%$ in our study. This was higher than the mortalities reported in previous studies [15-17] as well as in a document on haemovigilance published by the Japan Red Cross Society [14] that reported only four patients who may have died from TRALI from 2010 to 2015. In-hospital mortality in the matched control patients was as high as $16.7 \%$, suggesting the presence of critical clinical conditions in the patients in our cohort. The high mortality in our study can be interpreted as a consequence of the high mortality in the patient population examined.

Recently, risk factors for the development of TRALI have been extensively studied with respect to blood products used for transfusion [18]. The studies demonstrated the involvement of anti-HLA antibodies and/ or anti-human neutrophil antigen antibodies in the development of TRALI. Based on the results, use of these products has been avoided, resulting in a reduced incidence of TRALI [6].

Our results identified ILD as a risk factor for the development of TRALI. It could be argued that patients with TRALI may have been simultaneously diagnosed with ILD because TRALI cannot be easily distinguished from acute ILD exacerbation. To avoid such contamination in the present study, patients with ILD were defined as those who were diagnosed with ILD at admission.

The ICD-10 codes used to identify ILD included collagen vascular disease, hypersensitivity pneumonitis and idiopathic interstitial pneumonia. The common feature of patients with these diseases and patients with other pulmonary diseases evaluated in this study is chronic systemic inflammation. The production of cytokines that induce the inflammation associated with pulmonary diseases, such as interleukin (IL)-6 and IL-8, has been intensively studied [19-21]. A previous study on TRALI showed the involvement of these pro-inflammatory cytokines in the development of TRALI [5, 22, 23]. However, it remains unclear why 
ILD was the only pulmonary disease identified as a risk for the development of TRALI. Further studies exploring the mechanisms for the development of TRALI in patients with ILD are warranted to determine the differences among patients with various pulmonary diseases.

Limitations of the present study should be acknowledged. Firstly, we were unable to validate the diagnoses used in the study because the database did not contain the results of laboratory tests and imaging examinations, or medical records. Furthermore, a validation study to specifically evaluate the diagnosis of TRALI is unrealistic because of the rarity of the disease. However, although sensitivity of diagnoses was reported to be relatively low, the specificity of diagnoses was preserved in the database [24]. With regard to the diagnosis of TRALI, a great deal of effort has been devoted to the discrimination of TRALI from other conditions such as TACO and ARDS in previous studies [1, 25]. In the present study, diagnosis of TRALI was based solely on the judgement of the attending physicians. It may be possible that TRALI was contaminated with TACO or ARDS. However, considering that all three conditions are undesirable and serious conditions after blood transfusion, and particularly because TRALI and ARDS are pathophysiologically similar to one another, we think that the message of our study is not weakened by the contamination. Secondly, owing to a lack of data, we were unable to evaluate antibodies that can mediate TRALI in detail. Finally, because this was a retrospective study, the results are prone to bias owing to unmeasured confounders, such as high peak airway pressure while undergoing mechanical ventilation [5].

In conclusion, in this nested case-control study using a nationwide inpatient database in Japan, we showed that ILD was an independent risk factor for the development of TRALI. Physicians should be aware of the increased risk of TRALI in patients with ILD.

Provenance: Submitted article, peer reviewed

Conflict of interest: A. Yokoyama has nothing to disclose. Y. Sakamoto has nothing to disclose. T. Jo received consigned research funding from Tsumura \& Co. and belongs to a laboratory that is a joint programme with Tsumura \& Co. H. Urushiyama has nothing to disclose. H. Tamiya has nothing to disclose. G. Tanaka has nothing to disclose. H. Matsui has nothing to disclose. K. Fushimi has nothing to disclose. H. Yasunaga reports grants from Ministry of Health, Labour and Welfare, Japan, during the conduct of the study. T. Nagase has nothing to disclose.

Support statement: This work was funded by grants from the Ministry of Health, Labour and Welfare, Japan (H30-Policy-Designated-001, H30-Policy-Designated-004, and H29-ICT-Genral-004), and the Ministry of Education, Culture, Sports, Science and Technology, Japan (17H04141). The funding bodies had no role in the design of the study; collection, analysis, or interpretation of the data; or writing of the manuscript. Funding information for this article has been deposited with the Crossref Funder Registry.

References

1 Kleinman S, Caulfield T, Chan P, et al. Toward an understanding of transfusion-related acute lung injury: statement of a consensus panel. Transfusion 2004; 44: 1774-1789.

2 Vlaar APJ, Juffermans NP. Transfusion-related acute lung injury: a clinical review. Lancet 2013; 382: 984-994.

3 Gajic O, Rana R, Winters JL, et al. Transfusion-related acute lung injury in the critically ill: prospective nested case-control study. Am J Respir Crit Care Med 2007; 176: 886-891.

4 Vlaar APJ, Binnekade JM, Prins D, et al. Risk factors and outcome of transfusion-related acute lung injury in the critically ill: a nested case-control study. Crit Care Med 2010; 38: 771-778.

5 Toy P, Gajic O, Bacchetti P, et al. Transfusion-related acute lung injury: incidence and risk factors. Blood 2012; 119: $1757-1767$.

6 Schmickl CN, Mastrobuoni S, Filippidis FT, et al. Male-predominant plasma transfusion strategy for preventing transfusion-related acute lung injury: a systematic review. Crit Care Med 2015; 43: 205-225.

7 Silliman CC. The two-event model of transfusion-related acute lung injury. Crit Care Med 2006; 34: 5 Suppl., S124-S131.

8 Huertas A, Guignabert C, Barberà JA, et al. Pulmonary vascular endothelium: the orchestra conductor in respiratory diseases. Eur Respir J 2018; 51: 1700745.

9 Yasunaga H. The Diagnosis Procedure Combination database. Ann Clin Epidemiol 2019; 1: 76-79.

10 Woodward M. Epidemiology: study design and data analysis. 3rd Edn. Boca Raton, Chapman and Hall/CRC Texts in Statistical Science, 1999; pp. 265.

11 Silliman CC, Boshkov LK, Mehdizadehkashi Z, et al. Transfusion-related acute lung injury: epidemiology and a prospective analysis of etiologic factors. Blood 2003; 101: 454-462. 
12 Andreu G, Boudjedir K, Muller J-Y, et al. Analysis of transfusion-related acute lung injury and possible transfusion-related acute lung injury reported to the French Hemovigilance Network from 2007 to 2013. Transfus Med Rev 2018; 32: 16-27.

13 Okazaki H. TRALI-risk-reduction strategies: Japanese approach. ISBT Sci Ser 2011; 6: 422-426.

14 Japanese Red Cross Society. Haemovigilance by JRCS 2016. www.jrc.or.jp/mr/english/pdf/Haemovigilance2016 en.pdf Date last accessed: 9 October 2020

15 Wallis JP, Lubenko A, Wells AW, et al. Single hospital experience of TRALI. Transfusion 2003; 43: 1053-1059.

16 Breanndan Moore S. Transfusion-related acute lung injury (TRALI): clinical presentation, treatment, and prognosis. Crit Care Med 2006; 34: 5 Suppl.: S114-S117.

17 Popovsky MA. Transfusion and lung injury. Transfus Clin Biol 2001; 8: 272-277.

18 Mair DC, Hirschler N, Eastlund T. Blood donor and component management strategies to prevent transfusion-related acute lung injury (TRALI). Crit Care Med 2006; 34: 5 Suppl., S137-S143.

19 Ziegenhagen MW, Zabel P, Zissel G, et al. Serum level of interleukin 8 is elevated in idiopathic pulmonary fibrosis and indicates disease activity. Am J Respir Crit Care Med 1998; 157: 762-768.

20 De Lauretis A, Sestini P, Pantelidis P, et al. Serum interleukin 6 is predictive of early functional decline and mortality in interstitial lung disease associated with systemic sclerosis. J Rheumatol 2013; 40: 435-446.

21 Ziora D, Jastrzębski D, Adamek $M$, et al. Circulating concentration of markers of angiogenic activity in patients with sarcoidosis and idiopathic pulmonary fibrosis. BMC Pulm Med 2015; 15: 113.

22 Vlaar APJ, Hofstra JJ, Determann RM, et al. Transfusion-related acute lung injury in cardiac surgery patients is characterized by pulmonary inflammation and coagulopathy: a prospective nested case-control study. Crit Care Med 2012; 40: 2813-2820.

23 Roubinian NH, Looney MR, Kor DJ, et al. Cytokines and clinical predictors in distinguishing pulmonary transfusion reactions. Transfusion 2015; 55: 1838-1846.

24 Yamana H, Moriwaki M, Horiguchi H, et al. Validity of diagnoses, procedures, and laboratory data in Japanese administrative data. J Epidemiol 2017; 27: 476-482.

25 Gajic O, Gropper MA, Hubmayr RD. Pulmonary edema after transfusion: how to differentiate transfusion-associated circulatory overload from transfusion-related acute lung injury. Crit Care Med 2006; 34: 5 Suppl., S109-S113. 